\title{
Improvement of induction motor analysis accuracy in reluctance network analysis
}

\author{
Tomoyuki Umesaka*, Katsubumi Tajima*, and Yukihiro Yoshida** \\ *Cooperative Major in Life Cycle Design Engineering, Graduate School of Engineering and Resource Science, Akita Univ., \\ 1-1Tegatagakuen-machi, Akita 010-8502, Japan \\ ${ }^{* *}$ Department of Electrical and Electronic Engineering, Graduate School of Engineering and Resource Science, Akita Univ., \\ 1-1Tegatagakuen-machi, Akita 010-8502, Japan
}

In previous studies, we applied reluctance network analysis (RNA) to the dynamic analysis of the induction motor. However, the accuracy of our analysis of the motor characteristics using the RNA model was not high because the detailed magnetic flux distribution in the rotor was not taken into account. In this paper, we propose a 3D RNA model that considers the detailed magnetic flux distribution in the rotor and skew of the rotor bar. Using the proposed RNA model, the winding currents of the induction motor under no-load and locked rotor conditions can be calculated with higher accuracy than with the previous RNA model.

Keywords: Induction motor, Reluctance network analysis, Analysis of dynamic characteristics, Squirrel cage rotor, Skew, High-speed calculation

\section{Introduction}

In Japan, the proportion of thermal power to the total amount of power used has become large since most of the nation's nuclear power plants have ceased operation. The proportion of fossil energy to total energy in Japan was about $90 \%$ as of in 2011, and most of the fossil fuels were imported. It has been pointed out that the stable supply of future power in Japan will not be easy (1) because the power consumption of the world is increasing due to population growth and economic growth in emerging countries.

Therefore, improving the efficiency of motors used in various applications is strongly required. Of particular interest are induction motors, which are used in extensive applications ranging from large industrial equipment to compact household appliances.

To improve the efficiency of such motors, a high-precision calculation method of the motor characteristics of all potential materials and dimensions is required. The finite element method (FEM) has generally been used for electromagnetic devices in the past, but for dynamic analysis of the induction motor, a great deal of computing time and memory are required. Therefore, in a previous work the authors applied reluctance network analysis (RNA) to the dynamic analysis of the induction motor ${ }^{(2)}$.

In the current work, we present an analysis of the accuracy improvement of the dynamic characteristics of the induction motor based on RNA considering the detailed magnetic flux distribution and skew of the rotor.

\section{Capacitor motor (single-phase induction motor)}

\section{$\langle 2 \cdot 1\rangle$ Structure and principles of the capacitor motor}

The basic circuit of the capacitor motor is shown in Fig. 1, where $C_{r}$ is a running capacitor, $C_{s}$ is a starting capacitor, $v$ is the applied voltage, and $i_{m}$ and $i_{a}$ are the main winding and auxiliary winding currents, respectively.

By connecting $C_{r}$, the phase difference between the auxiliary and main winding currents is almost 90 degrees. As a result, the rotating magnetic field is generated around the squirrel cage rotor, and the motor generates a driving torque.

\section{$\langle 2 \cdot 2\rangle$ Specifications of analysis object}

The test motor is a capacitor start-type capacitor run motor (SKD-DBKK8) made by Toshiba. Its specifications and dimensions are listed in Table 1 . The main winding and auxiliary winding are both distributed windings. Figure 2 and Table 2 show the arrangement and number of turns, respectively ${ }^{(2)}$.

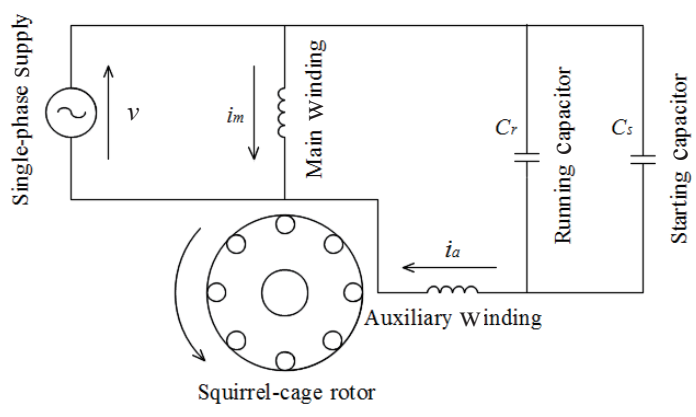

Fig. 1 Circuit configuration of a capacitor motor. 


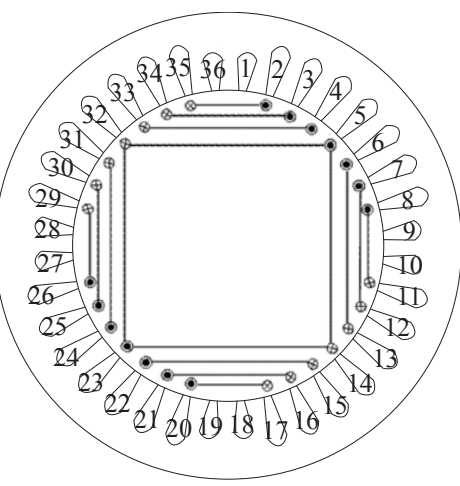

(a) Main windings

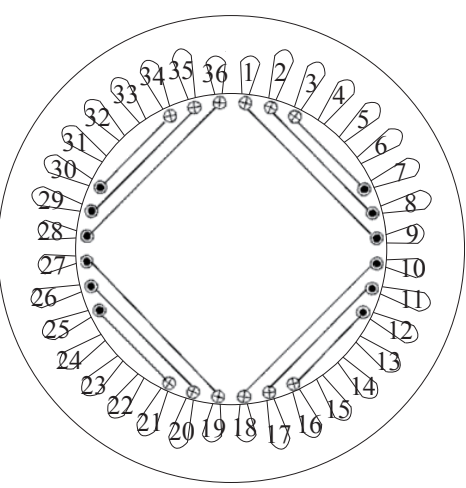

(b) Auxiliary windings

Fig. 2 Arrangement of main windings.

Table 1 Specifications of specimen motor.

\begin{tabular}{|c|c|}
\hline Parameter & Value \\
\hline Frequency & $50 \mathrm{~Hz}$ \\
\hline Voltage & $100 \mathrm{~V}$ \\
\hline Current & $12.6 \mathrm{~A}$ \\
\hline Output & $750 \mathrm{~W}$ \\
\hline Number of poles & 4 \\
\hline Rated speed & $1440 \mathrm{rpm}$ \\
\hline Running capacitor & $40 \mu \mathrm{F}$ \\
\hline Starting capacitor & $350 \mu \mathrm{F}$ \\
\hline Number of stator slots & 36 \\
\hline Internal diameter of stator & $90.0 \mathrm{~mm}$ \\
\hline Outer diameter of stator & $146.0 \mathrm{~mm}$ \\
\hline Gap width & $0.3 \mathrm{~mm}$ \\
\hline Number of rotor slots & 44 \\
\hline Outer diameter of rotor & $89.4 \mathrm{~mm}$ \\
\hline Iron core length & $93.0 \mathrm{~mm}$ \\
\hline
\end{tabular}

Table 2 Number of turns of windings.

\begin{tabular}{|c|c|c|c|}
\hline \multicolumn{2}{|c|}{ Main windings } & \multicolumn{2}{c|}{ Auxiliary windings } \\
\hline \hline Slot number & Turns & Slot number & Turns \\
\hline $35-2,11-8,17-20,29-26$ & 7 & $1-9,18-10,19-27,36-28$ & 36 \\
\hline $34-3,12-7,18-21,30-25$ & 11 & $2-8,17-11,20-26,35-29$ & 18 \\
\hline $33-4,13-6,15-22,31-24$ & 14 & $3-7,16-12,21-25,34-30$ & 5 \\
\hline $32-5,14-5,14-23,32-23$ & 7 & & \\
\hline
\end{tabular}

\section{2-dimensional RNA model of capacitor motor}

\section{$\langle 3 \cdot 1\rangle \quad$ Overview of Reluctance Network Analysis}

The authors previously proposed a method of reluctance network analysis (RNA) that is suitable for dynamic simulations of an orthogonal-core type variable inductor, the SR motor and IPM motor, because of its simple modeling, high calculation accuracy, and ease of coupled analysis. Moreover, in calculation based on RNA, it is possible to use a general-purpose circuit simulation program such as SPICE.

We explain the flow of construction of the RNA model of the capacitor motor below.

\section{$\langle 3 \cdot 2\rangle$ RNA model of stator ${ }^{(3)}$}

The cross section of the capacitor motor is roughly composed of a stator, a rotor, and the air gap, as shown in Fig. 3.

Because the stator has 36 slots in a circumferential direction, we divide it into 36 parts in the circumferential direction, as shown in Fig. 4. The division of each part includes the surrounding space so as to consider the linkage fluxes into multiple elements, as shown in Fig. 5. In the figure, each tooth is divided into two equal parts because the concentrated magnetomotive forces due to winding current are arranged as shown in Fig.5. The divided elements can be expressed by a unit two-dimensional magnetic circuit, as shown in Fig. 6.

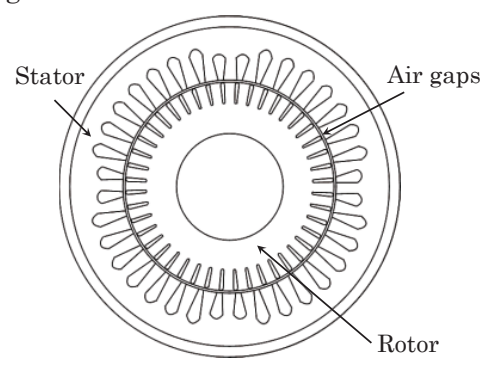

Fig. 3 Cross section of a capacitor motor.

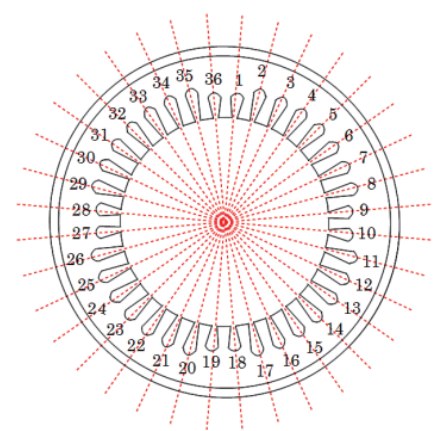

Fig. 4 Division in the radial direction of stator. 


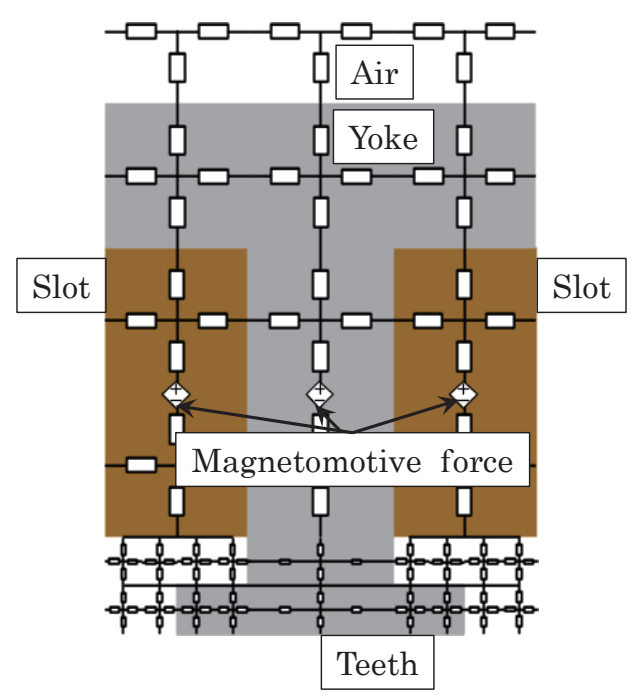

Fig. 5 RNA model of the stator (1/36).

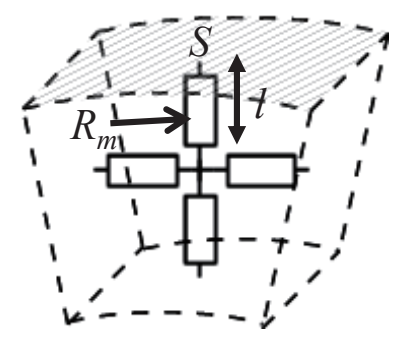

Fig. 6 Magnetic circuit of element.

Reluctance $R_{\mathrm{m}}$ in this unit magnetic circuit can be represented by equation (1) with magnetic permeability $\mu$, magnetic path length $I[\mathrm{~m}]$, and sectional area $S\left[\mathrm{~m}^{2}\right]$ :

$$
\boldsymbol{R}_{\boldsymbol{m}}=\frac{l}{\mu \mathrm{S}}
$$

Magnetomotive force is proportional to the number of turns $N$ and current $i$ of the coil. The magnetomotive force source is placed in consideration of the distributed winding of the main and auxiliary winding between stator slots ${ }^{(2)}$

\section{$\langle 3 \cdot 3\rangle$ RNA model of the rotor ${ }^{(3)}$}

The rotor of the capacitor motor used in this paper is the squirrel cage rotor. It has 44 slots in the circumferential direction, so we divide the rotor into 44 parts in the circumferential direction, as shown in Fig. 7, where each part is represented as a magnetic circuit network, as shown in Fig. 8. Therefore, by linking 44 circuits, we can derive the RNA model of the rotor.
The rotor has a chain structure consisting of end rings and conductor bars. The magnetic flux $\phi$ of the stator flows to the rotor and the electromotive force $e$ is generated by Faraday's law of electromagnetic induction in the rotor to flow the eddy current $i$. In addition, according to the law of Lenz, eddy current $i$ acts to prevent the change of the magnetic flux flow, and generates a magnetomotive force in the opposite direction of the magnetic flux. We obtained a chain electrical circuit composed of 44 circuits (Fig. 9), where the size of the conductor bar is $R_{\text {bar }}=1.14 \times 10^{-4} \Omega$, the size of the end ring is $R_{\text {end }}=4.54 \times 10^{-6} \Omega$, and the resistivity is $75^{\circ} \mathrm{C}$ of aluminum.

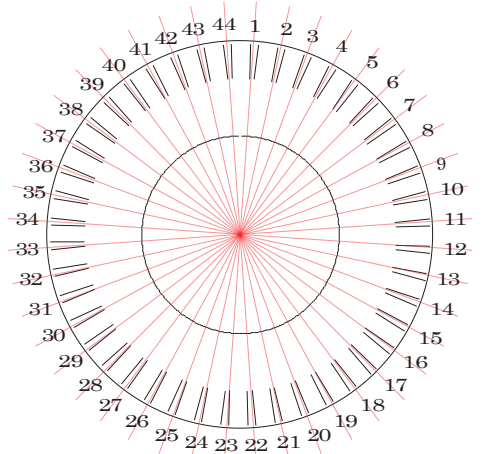

Fig. 7 Division in the radial direction of the rotor.

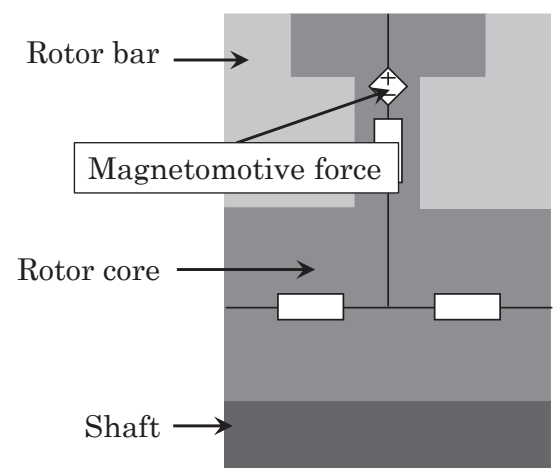

Fig. 8 RNA model of the rotor (1/44).

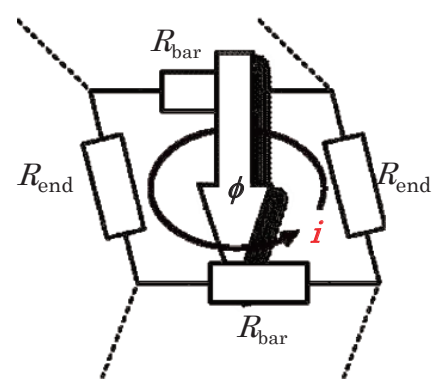

Fig. 9 Electric circuit model of the rotor. 


\section{$\langle 3 \cdot 4\rangle$ Driving expression of the rotor ${ }^{(4)}$}

In an earlier work ${ }^{(5)}$, we proposed an RNA model of the rotor when the rotor is driving. This model consists of an electrical circuit to evaluate the induced currents on the rotor bars and end rings and a magnetic circuit to express the MMFs corresponding the induced currents on the rotor, as shown in Fig. 10. In the figure, there are eight stator slots and eight rotor slots .

The flux flowing into the first slot of the rotor $\phi_{11}$ shown in Fig. 10 (a) is obtained by

$$
\phi_{r 1}=\frac{4}{\pi} \cdot\left\{\left(\frac{\pi}{4}-\theta\right) \phi_{s 1}+\theta \cdot \phi_{s 2}\right\}\left(0 \leq \theta \leq \frac{\pi}{4}\right),
$$

where $\theta$ is the rotating angle of the rotor. The magnetomotive force generated by the induced current in the rotor electric circuit $M M F_{1}$ shown in Fig. 10 (b) is expressed by

$$
M M F_{1}=\frac{4}{\pi} \cdot\left\{\left(\frac{\pi}{4}-\theta\right) i_{r 1}+\theta \cdot i_{r 8}\right\}\left(0 \leq \theta \leq \frac{\pi}{4}\right),
$$

where $i_{\mathrm{r} 1}$ and $i_{18}$ are the electric currents calculated by the electric circuit model.

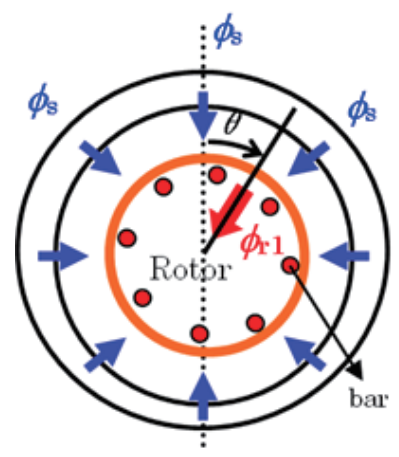

(a) Fluxes flowing into the rotor.

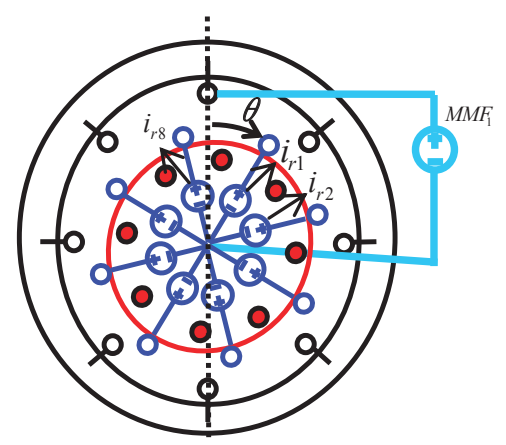

(b) MMFs acting on the stator

Fig. 10 Expression of coupling between the rotor and stator.

\section{3-dimensional RNA model ${ }^{(5)}$}

\section{$\langle 4 \cdot 1\rangle$ New RNA model of the rotor}

As described in $\langle 3.3\rangle$, the previous RNA model of the rotor in Fig. 8 is a reverse T-shaped simple magnetic circuit that did not consider the magnetic flux distribution of the core and skew of the rotor bars. To improve the analysis accuracy of the motor, we have developed a new RNA model that represents the flux distribution in the rotor and skew. We divide a part of the rotor shown in Fig. 8 into 19 elements corresponding to the shape of the conductor bar and the iron core of the rotor to make the new RNA model.

\section{$\langle 4 \cdot 2\rangle$ Driving expression of new RNA model}

Here, we consider the magnetic flux $\phi_{r}$ passing through the rotor core. In the previous model (Fig. 8), all $\phi_{r}$ passed through the rotor core. In contrast, in the model proposed here (Fig. 11), $\phi_{r}$ flows in both the rotor core and the conductor bar. In other words, $\phi_{r}^{\prime}$ passes through the rotor core between the conductor bars.

We calculate $\phi_{r}^{\prime}$ on the rotating state as follows.

(1) Flux $\phi_{r}$ can be given by using Fig. 10 (a): it flows from the stator to $1 / 44$ of the rotor model (Fig. 11).

(2) However, the magnetic flux $\phi_{r}^{\prime}$ flowing between the conductor bars is different from $\phi_{r}$. For this reason, we use another RNA model to calculate $\phi_{r}^{\prime}$ from $\phi_{r}$ (Fig. 12).

(3) To obtain a reaction field magnetomotive force $i_{r}$ in the electric circuit model of the rotor using the $\phi_{r}$, we give the original magnetic circuit $M M F_{1}-M M F_{44}$ with reference to Fig. 10. (b).

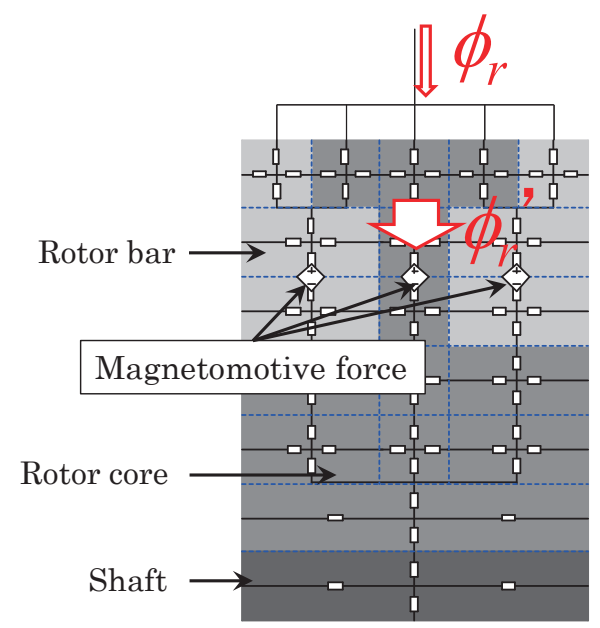

Fig. 11 New RNA model of the rotor(1/44). 


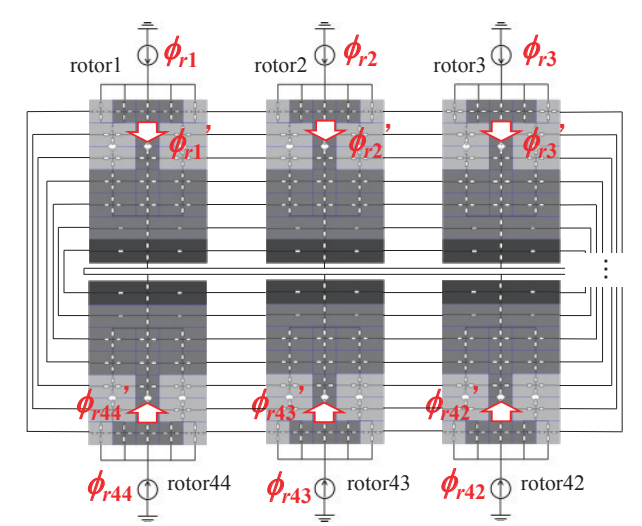

Fig. 12 Calculation circuit of $\phi_{11}{ }^{\prime}-\phi_{144}$, from $\phi_{11}-\phi_{144}$.

\section{$\langle 4 \cdot 3\rangle$ Consideration of skew}

The conductor bars of the squirrel-cage rotor of the induction motor are skewed as shown in Fig. 13. Because the 2-dimensional RNA model cannot express the skew, a 3 -dimensional model is needed to consider the axial direction structure. In this paper, we constructed a 3-dimensional RNA model representing the rotor structure of the axial direction to consider the skew of two slots. The rotor bar shape was approximated using a three-layer structure as shown in Fig. 14. The 3 -dimensional unit magnetic circuit used is shown in Fig. 15. For the laminated structure of the rotor core, the reluctance $R^{\prime}$ in the laminated direction was calculated as a relative permeability of 25 using the methods of reference 6 .

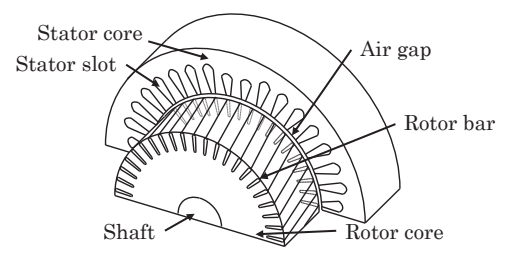

Fig. 13 Stator and rotor of capacitor motor.

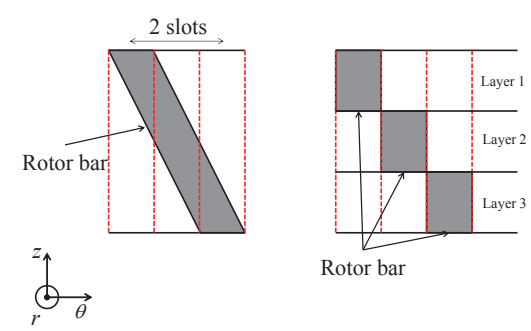

Fig. 14 Consideration of skew for the RNA model.

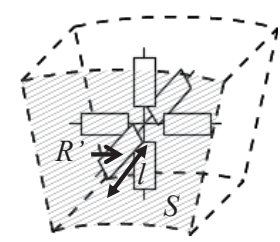

Fig. 15 3-dimensional magnetic circuit of element.

\section{Results and discussion}

We examined the validity of the new RNA model of the capacitor motor by using the OrCAD PSpice 16.6 (Cadence Design Systems, Japan) as a solver for the analysis and a regular PC (Intel®Core (TM) i7-3770KCPU @ $3.5 \mathrm{GHz} 3.5 \mathrm{GHz} 16.0$ GB RAM). Because the same flux distribution appears at every 180 degrees in the four-pole structure, we use a half model.

With the stator and rotor core material, we assume a non-oriented silicon steel sheet 50H600 (NIPPON STEEL CORPORATION) and a relative magnetic permeability of 4000 . The rotor conductor bars were the same relative permeability as air because their material is non-magnetic.

In the case of applying the sinusoidal voltage of $50 \mathrm{~Hz}$, the waveforms of the currents in the previous and proposed model with locked rotor and under no-load are shown in Figs. 16 and 17. As we can see, the calculation accuracy is improved. Moreover, from Fig. 17 shows that the current pulsation occurring the previous model is reduced. This pulsation is thought to stem from errors in the magnetic flux and the magnetomotive forces of the linear interpolation for use in driving expression, as described in $\langle 3.4\rangle$. However, by considering the detailed magnetic flux distribution and the skew, we are able to reduce the magnitude of the pulsation.

The effective values $I_{\mathrm{m}}, I_{\mathrm{a}}$ obtained from these current waveforms are shown in Tables 3 and 4. Calculation errors of each winding current are compared with the calculation results by the previous model. Results show that they are greatly reduced. In particular, the calculation error of the main winding current of the motor with locked rotor is reduced.

Figure 18 shows the change of $I_{\mathrm{m}}$, and $I_{\mathrm{a}}$ to slip $s$. As we can see in figure, there is good agreement around the synchronous speed. However, the error of the main winding current is increased with the increase of slip $s$.

Twenty cycles of computation took about 30 minutes with the locked rotor, and about one hour in the rotational state. 


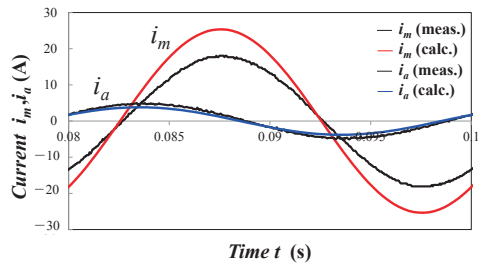

(a) Previous model

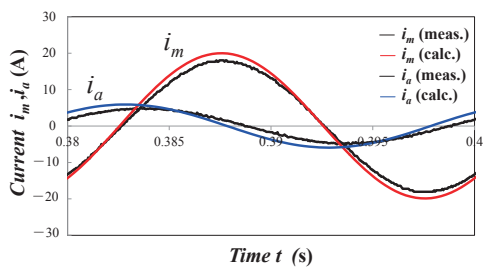

(b) Proposed model

Fig. 16 Current waveforms of the motor with locked rotor.

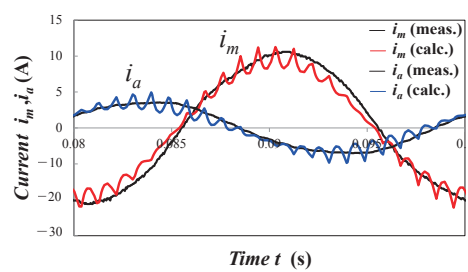

(a) Previous model

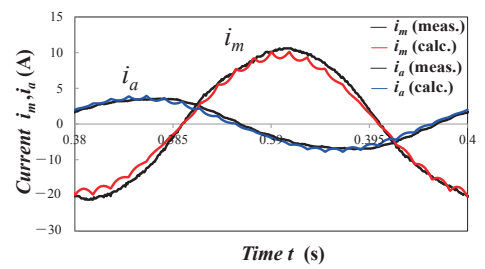

(b) Proposed model

Fig. 17 Current waveforms of the motor under no-load.

Table $3 I_{m}$ and $I_{a}$ of the motor with locked rotor.

\begin{tabular}{|c|c|c|c|c|}
\hline \multicolumn{5}{|c|}{ (Input voltage $25.6\left[\mathrm{~V}_{\mathrm{rms}}\right]$ ) } \\
\hline & \multicolumn{2}{|c|}{$I_{m}\left[\mathrm{~A}_{\mathrm{rms}}\right]$} & \multicolumn{2}{|c|}{$I_{a}\left[\mathrm{~A}_{\mathrm{rms}}\right]$} \\
\hline & previous & proposed & previous & proposed \\
\hline Measurement & \multicolumn{2}{|c|}{12.6} & \multicolumn{2}{|c|}{3.45} \\
\hline Calculation & 18.1 & 14.4 & 2.68 & 4.11 \\
\hline Relative error & $43.7 \%$ & $14.3 \%$ & $22.3 \%$ & $19.1 \%$ \\
\hline
\end{tabular}

Table $4 I_{m}$ and $I_{a}$ of the motor under no-load.

\begin{tabular}{|c|c|c|c|c|}
\multicolumn{2}{c|}{ (Input voltage $100\left[\mathrm{~V}_{\mathrm{rms}}\right]$ ) } \\
\hline & \multicolumn{2}{|c|}{$I_{m}\left[\mathrm{~A}_{\mathrm{rms}}\right]$} & \multicolumn{2}{c|}{$I_{a}\left[\mathrm{~A}_{\mathrm{rms}}\right]$} \\
\hline & previous & proposed & previous & proposed \\
\hline Measurement & \multicolumn{2}{|c|}{7.35} & \multicolumn{2}{c|}{2.57} \\
\hline Calculation & 6.45 & 6.99 & 2.66 & 2.56 \\
\hline Relative error & $12.2 \%$ & $4.9 \%$ & $3.5 \%$ & $0.4 \%$ \\
\hline
\end{tabular}

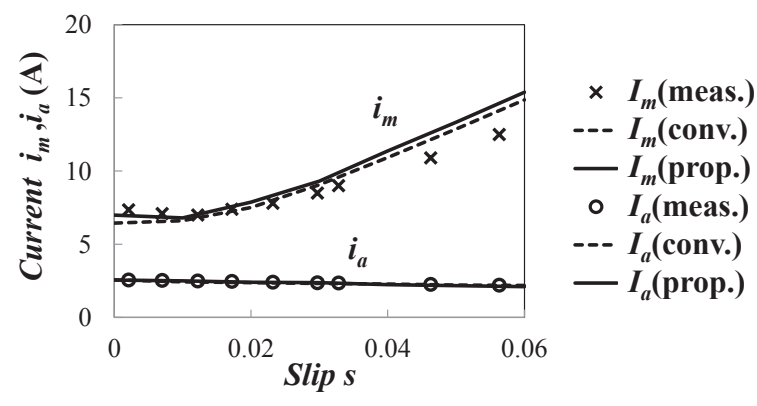

Fig. 18 Load characteristics of motor.

\section{Conclusions}

We proposed a 3D RNA model that considers the detailed magnetic flux distribution in the rotor and skew of the rotor bar. Experimental results showed that the calculation accuracy of the winding currents under no-load and locked rotor conditions are improved compared with the previous RNA model. Also, the pulsation of winding current waveforms is reduced while maintaining high-speed calculation. This is much-needed progress in the high-precision characteristic calculation of the induction motor.

However, the calculation error of the main winding current of the motor with load became large in the low speed area. Therefore, in our future work we intend to improve the accuracy of the load characteristics of the induction motor to apply the proposed method to induction motor design.

\section{References}

(1) Ministry of Economy, Trade and Industry Agency for Natural Resources and Energy: "2013 fiscal year The annual report on energy (Energy White Paper 2014)”, pp. 179-218 (2010).

(2) K. Tajima and T. Sato, J. Magn. Soc. Jpn., Vol. 34, No. 3, pp. 367-373 (2010).

(3) T. Miyaji, K. Tajima, T. Taniguchi and T. Sato, J. Magn. Soc. Jpn., Vol. 27, No. 9, pp. 976-981 (2003).

(4) K. Tajima, M. Hattori, T. Miyaji, T. Sato, and Y. Sakamoto, J. Magn. Soc. Jpn., Vol. 29, No. 6, pp. 680-685 (2005).

(5) T. Umesaka, K. Tajima, and Y. Yoshida, The papers of Technical Meeting on Magnetics, IEE Jpn, MAG-15-116 (2015).

(6) S. Hayakawa, K. Nakamura, S. Akatsuka, T. Aoki, M. Kawakami, T. Ohinata, K. Minazawa, and O. Ichinokura, J. Magn. Soc. Jpn. 28, 425 (2004)

Received Nov. 16, 2015; Revised Feb. 29, 2016; Accepted Mar. 21,2016 